\title{
Genetic Variation of Eight Indonesian Swamp-Buffalo Populations Based on Cytochrome b Gene Marker
}

\author{
M. Rusdin ${ }^{\mathrm{a}, \mathrm{b}}$, D.D. Solihin' ${ }^{\mathrm{c}}$ A. Gunawan ${ }^{\mathrm{d}}$, C. Talib $^{\mathrm{e}}$, \& C. Sumantri ${ }^{\mathrm{d}, *}$ \\ ${ }^{a}$ Graduate School, Animal Production and Technology Study Program, IPB University (Bogor Agricultural University) \\ ${ }^{b}$ Department of Animal Science, Faculty of Animal Science, Halu Oleo University \\ Kampus Hijau Bumi Tridharma, Jalan H.E.A. Mokodompit, Anduonohu, Kendari, Sulawesi Tenggara 93232, Indonesia \\ 'Department of Biologi, Faculty of Mathematic and Natural Science, IPB University (Bogor Agricultural University) \\ ${ }^{\mathrm{d} D e p a r t m e n t}$ of Animal Production and Technology, Faculty of Animal Science, IPB University \\ (Bogor Agricultural University) \\ Kampus IPB Darmaga Bogor 16680, Indonesia \\ eIndonesian Center for Animal Research and Development \\ Jalan Raya Pajajaran Kav. E-59, Bogor 16151, Indonesia \\ ${ }^{*}$ Corresponding author: csumantri12@gmail.com \\ (Received 20-05-2019; Revised 06-08-2019; Accepted 11-09-2019)
}

\begin{abstract}
Genetic variation is a major concern in animal genetic resources conservation program. This study aimed to analyze genetic variation and phylogeography of eight Indonesian swamp-buffalo populations based on cytochrome $b$ gene marker. A total of 78 DNA fragment samples originating from eight Indonesian swamp-buffalo populations were used in this study, namely Bombana Island, Bombana mainland, Kolaka, Konawe, North Toraja, West Nusa Tenggara, Banten, and Aceh with 11, $10,13,14,10,10,5$, and 5 samples, respectively. The cytochrome $b$ gene sequence and genetic variation parameters were analyzed in MEGA software (ver 6), and DnaSP software (ver 5.10.01). The results of this study showed that all DNA-fragment samples were successfully amplified by PCR technique with the size target $(906 \mathrm{bp}$ ). Based on the distribution of all samples, it was found 9 polymorphic sites, and 10 haplotypes with the haplotype diversities were 0.6590 . The average of genetic distances between populations ranged from $0.0000-0.002$. They were grouped into two main clusters. The first cluster consisted of Aceh, North Toraja, West Nusa Tenggara, Banten, Kolaka, and Konawe populations, meanwhile, the second cluster consisted of Bombana Island, Bombana mainland, Kolaka, and Konawe populations. The results of the study were concluded that eight Indonesian local swamp-buffalo populations were grouped into two main clusters where Bombana Island and Bombana mainland populations were specific breeds because they were only found in the second cluster and also had specific nucleotides sites on the 57 nucleotides which $C$ base changed to $T$. The results of this study were useful in formulating the program of conservation and utilization of Indonesian buffalo genetic resources, especially in the buffalo population with specific breeds.
\end{abstract}

Keywords: cytochrome b; local swamp buffalo; polymorphic sites; specific nucleotides

\section{INTRODUCTION}

The buffalo population has decreased drastically in almost all countries in Southeast Asia. By the years of 1990-2016 period, for example, the decreasing buffalo populations were successively for the highest in Thailand $(82.59 \%)$, followed by Indonesia $(58.43 \%)$, and Malaysia (42.19\%), meanwhile the Philippines has slightly increased (3.58\%) (FAOSTAT 2015; FAOSTAT 2018). The total population and productivity of buffaloes in Indonesia were much lower than that of cattle. In 2018, the local buffalo population in Indonesia was $1,356,390$ heads; meanwhile the cattle population was $17,050,006$ heads. The potency of buffaloes as national meat resources was only $0.88 \%$; meanwhile the potency of cattle was 13.81\% (Ditjen of PKH, 2018). The trend of population declining is a serious threat to the sustainability of buffalo genetic resources.
The buffalo population in Indonesia, almost 100\% are swamp buffaloes, meanwhile river buffalo are less than $1 \%$ and only develops in North Sumatra (Talib et al., 2014). Swamp buffaloes are spreaded in almost all regions in Indonesia because they have high adaptability. Recently, there have been seven of location-specific buffalo breeds in Indonesia declared by the Decree of the Minister of Agriculture, namely Pampangan buffalo (South Sumatera), Simelue buffalo (Nanggroe Aceh Darussalam), Moa buffalo (Maluku), Sumbawa buffalo (West Nusa Tenggara), Toraya buffalo (South Sulawesi), South Kalimantan buffalo, and East Kalimantan buffalo (Talib et al., 2014; Sumantri et al., 2017). The local breeds of buffaloe have been adapted very well in their specific environments. The buffalo of Pampangan, South Kalimantan, and East Kalimantan adapt to the environment of swamps, lakes, and rivers. Sumbawa and Moa buffaloes adapt to savanna areas with droughts that 
are more than eight months per year. Simelue buffaloes adapt to coastal and hilly areas, meanwhile Toraya buffaloes adapt to agricultural areas (Talib et al., 2014).

Mitochondrial DNA marker has been used as one approach in molecular genetic studies to identify genetic variation and phylogeny in the animal. The main reason for using mitochondrial DNA marker because the mitochondrial genome has a relatively small size $( \pm$ $16500 \mathrm{bp}$ ), and a rapid evolutionary rate, which causes high intra-species diversity (Avise, 1994). Mammalian mitochondrial DNAs show several special features such as an absence of intron, maternal inheritance, all offsprings from the same female parent having the same mitochondrial DNA sequence, the existence of single copy of orthologous genes, the lack of recombination events and a high mutation rate (Linacre \& Tobe, 2011; Kim et al., 2013). Mitochondrial DNAs of the animal contain thirteen protein-coding genes and are considered as strong markers for genetic variation analysis such as at the family, genus, and species levels (Arif \& Khan, 2009; Doosti et al., 2014). The cytochrome b (Cyt b) gene is one of the protein-coding genes in mitochondrial containing abundant phylogenetic information among intra-species and inter-species and it is considered to be a good marker to study the genetic differentiation and phylogenetic relationships among species within the same genus or the same family. Cyt $b$ gene markers have been proved as an efficient and powerful tool for breed characterization and species identification (Browers et al., 1994; Zardoya \& Meyer, 1996; Saif et al., 2012).

Previous studies on genetic variation and phylogeography based on mitochondrial Cyt b gene marker in livestock have been reported, such as in goats (Pakpahan et al., 2016), Romanian cattle (Xuan et al., 2010), Korean cattle (Kim et al., 2013), Pakistani cattle (Hussain et al., 2018), Indonesian local cattle (Hartatik et al., 2015; Hartatik et al., 2018), Nili-Ravi and Kundi buffaloes in Pakistan (Saif et al., 2012), and Southeast Asian buffaloes (Lau et al., 1998; Yindee et al., 2010; Lei et al., 2011; Zhang et al., 2011; Zhang et al., 2016). Study related to genetic variation and genetic relationships of Indonesian local swamp buffaloes was reported by Sukri et al. (2014) that from eight Indonesian local swamp-buffalo populations (Madiu, Aceh, Blitar, Riau, Kalimantan, Tator, Bali, Lombok, and Bima), it was found $19.87 \%$ variable sites consisted of 16 haplotypes and 7 different haplogroups unique based on their geographical regions. Meanwhile, Amin et al. (2015) reported that four local swamp-buffalo populations in Central Indonesia (Bali, Toraja, Lombok, and Bima) had close genetic relationships, which they only consisted of one main cluster and two sub-clusters. Also, there are still many local swamp buffaloes from the other regions in Indonesia that have unique characteristic with specific geographical spread, such as swamp buffaloes from Banten, Riau, Sumenep, and Southeast Sulawesi (Sumantri et al., 2017), but their genetic variations and genetic relationships have not been identified yet.

Information about genetic variation within and between population or breed has been needed in conservation and breeding programs of buffalo. The data of genetic differences and genetic relationships between and among populations or breeds are important to determine the best approach of AnGR conservation (Zhang et al., 2011). Therefore, this study was carried out to analyze the genetic variation and phylogeography of eight Indonesian swamp-buffalo populations based on mitochondrial Cyt b gene marker.

\section{MATERIALS AND METHODS}

\section{Sample Collection and Genomic DNA Extraction}

A total of 78 blood samples of local swamp-buffalo from eight different populations were used in this study (Table 1). Blood samples (3 mL) were collected from jugular veins and put in vacutainer tubes containing EDTA, then absolute ethanol was added and the mixture was stored at $4^{\circ} \mathrm{C}$. Genom DNAs were extracted from the blood. The Genomic DNA extraction protocol was performed according to Sambrook \& Russel (2001) with a minor modification.

\section{Primer Design}

The forward and reverse primers were designed using the Primer3 software (ver 4.0) (http://primer3. ut.ee) based on the Cyt $b$ gene sequence of swamp buffalo from GenBank with acc. No. D88637. The primer forward was for: 5'- CAT TCA TTG ACC TCC CTG CT $-3^{\prime}$, and the reverse primer was for $5^{\prime}$ - GCC GGA ACA TCA TAC TTC GT $-3^{\prime}$.

\section{DNA Amplification}

The DNA fragment was amplified using the Polymerase Chain Reaction (PCR) technique using a thermocycler machine (GeneAmp® PCR System 9700, Applied Bio SystemTM, Foster City, CA, USA). DNA amplification was carried out at a total volume of 50 $\mu \mathrm{L}$, containing $50 \mathrm{ng} / \mu \mathrm{L}$ DNA templates, 25 pmol primers (IDT, Singapore), 1 unit of Go Taq ${ }^{\circledR}$ Green Master Mix (Promega, Madison, WI, USA), and water. The PCR process was run with 30-35 cycles consisting of predenaturation at $95^{\circ} \mathrm{C}$ for $5 \mathrm{~min}$, denaturation at $95^{\circ} \mathrm{C}$ for $10 \mathrm{~s}$, annealing at $52^{\circ} \mathrm{C}$ for $20 \mathrm{~s}$, extension at $72^{\circ} \mathrm{C}$ for $30 \mathrm{~s}$, and the final extension at $72^{\circ} \mathrm{C}$ for $5 \mathrm{~min}$. The PCR products were visualized through $1.5 \%$ agarose

Table 1. Number of blood samples of local swamp buffalo based on population

\begin{tabular}{lc}
\hline Populations & Total (n) \\
\hline Bombana Island (Southeast Sulawesi 1) & 11 \\
Bombana Mainland (Southeast Sulawesi 2) & 10 \\
Kolaka (Southeast Sulawesi 3) & 13 \\
Konawe (Southeast Sulawesi 4) & 14 \\
North Toraja (South Sulawesi) & 10 \\
Mataram (West Nusa Tenggara) & 10 \\
Lebak (Banten) & 5 \\
Aceh (Nanggroe Aceh Darussalam) & 5 \\
Total (n) & 78
\end{tabular}


gel electrophoresis (V/W) which was stained with FluoroSafe DNA Staining ( $1^{\text {st }}$ Base, Singapore) above UV Transilluminator machine (Alpha Imager, Alpha Innotech, Santa Clara, USA).

\section{Sequence Analysis}

PCR products were sequenced using the Sanger method through analysis services from the 1st Base, Malaysia. All sequence results (ABI trace files) were edited in FinchTV software (ver 1.4.0) (www.geospiza. com). The Basic Local Alignment Search Tool (BLAST) was used to identify the similarity (homology) with Cyt b gene sequences in GenBank (https://blast.ncbi. nlm.nih.gov). Sequence analysis was performed using the Multiple Sequence Alignment Analysis Technique by using the Clustal program in MEGA software (ver 6) (Tamura et al., 2013). Cyt b gene sequence from GenBank with acc. No. D88637 was used as a reference. Cyt $b$ gene sequence that was analyzed did not include sequences of forward and reverse primers with base length was $866 \mathrm{bp}$.

\section{Data Analysis}

Variables observed included conservation sites, variable sites, transition/transversion substitutions, and genetic distances were then analyzed by MEGA software (ver 6) (Tamura et al., 2013). The phylogenetic tree was reconstructed based on the Neighbor-Joining method with 1000 bootstraps. The haplotype diversity was analyzed by DnaSP software (ver 5.10.01) (Rozas et al., 2010). The Network software (ver 5.0) (Available at http://www.Fluxux-engineering.com) was used to reconstruct a median-joining network of haplotypes.

\section{RESULTS}

\section{DNA Amplification and Sequence Analysis}

A total of 78 DNA fragment samples in this study were successfully amplified using PCR (Polymerase Chain Reaction) technique. The size of PCR product target was $906 \mathrm{bp}$. Bands pattern visualization of Cyt b fragment in 1.5\% agarose gel electrophoresis is presented in Figure 1, and the primers annealing position is presented in Figure 2. The BLAST results showed that the similarity percentage of Cyt $b$ gene sequences was at the range of $99-100 \%$ with query cover percentage was $100 \%$.

\section{Nucleotide Variation}

Nucleotide variations of the Cyt $b$ genes of eight Indonesian swamp-buffalo populations are presented in Table 2. The results of multiple alignment analysis of $78 \mathrm{Cyt} b$ genes sequences of Indonesian swamp buffalo were found $857(98.96 \%)$ conserved sites and 9 (1.04\%) variable (polymorphic) sites. The highest variable sites were found 5 sites $(0.8 \%)$ in Konawe and Bombana Island populations; meanwhile the lowest one was found 2 sites $(0.23 \%)$ in North Toraja, NTB, and Banten populations. Nucleotides position at variable sites of swamp buffalo Cyt b genes is presented in Figure 3.

Variable sites consisted of $3(0.35 \%)$ parsimony sites-informative and $6(0.69 \%)$ singleton sites. A site is parsimony-informative if it contains at least two types of nucleotides, and at least two of them occur with a minimum frequency of two; meanwhile a singleton site contains at least two types of nucleotides with, at most, one occurring multiple times (Tamura et al., 2013). The highest parsimony site was found 3 sites $(0.35 \%)$ in

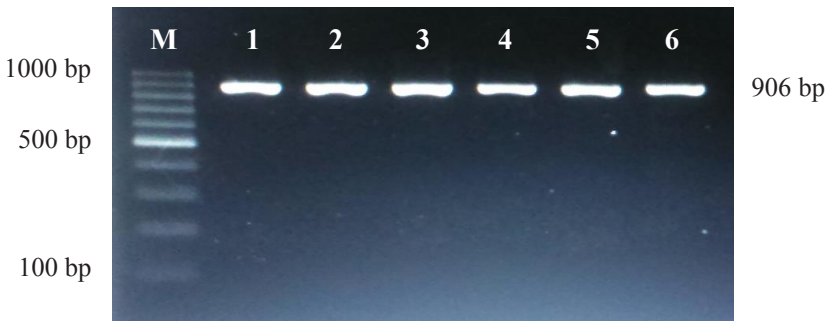

Figure 1. The band pattern visualization of PCR products of Cyt $\mathrm{b}$ gene of local swamp buffaloes in $1.5 \%$ agarose electrophoresis. $\mathrm{M}=$ marker, number $1-6=$ samples of PCR product.

\footnotetext{
1 atgaccaaca tccgaaaatc ccacccacta ataaaattc taaacaatgc attcattgac 4 Forward

61 ctccetgctc catcaaacat ctcatcatga tgaaactttg gctctctcct aggcatctgc

121 ctaatcctgc aatcctcac cggcctattc ctagcaatac actacacatc cgacacaaca

181 acagcattct cctccgtcgc ccacatctgc cgggacgtga actatggatg aattattcga

241 tacatacacg caaacggagc ttcaatattt ttcatctgct tatatataca cgtaggacga

301 ggcatatact acggatcata taccttcta gaacatgaa acatcggagt aatcctacta

361 ttcgcagtaa tagccacagc atttatagga tacgtactgc catgaggaca aatatcattc

421 tgaggggcaa cagtcatcac caaccttctg tcagcaatcc catacattgg tacaagtctg

481 gttgaatgaa tttgaggggg attctcagta gacaaagcaa ccctcacccg attcttcgca

541 tttcacttca tcctcccatt cattatcgca gcacttgcaa tagtccacct attattetc

601 cacgaaacag gatccaacaa cccaacagga atctcatcag acacagacaa aatcccattc

661 cacccctatt acaccattaa agacatccta ggcgccctac tattaatcct agccctaata

721 ctattagtac tattcgcacc cgacctcctc ggggacccag acaactacac cccagcaaac

781 ccactcaaca cacctcccca catcaagcct gaatggtact tcctattcgc atacgcaatc

841 ttacgatcaa ttcctaacaa actaggaggg gttctagccc tagttctctc tatcctaatc

901 ctcattctca tgcccctgct acatacatcc aaacaacgaa gtatgatgtt ccggccattc $\leftarrow$ Reverse

961 agccaatgcc tattctgaat tctagtagca aacctgctaa cactcacatg gattggagga

1021 cagccagtcg aacacccata tattatcatt ggacaactag catctatcac atattccttc

1081 ctcatcctag tgttaatacc aacggccagc ataatcgaaa ataatctctt aaatgaaga
}

Figure 2. The position of primers in a complete sequence of Cyt b gene of Bubalis bubalis carabanensis from GenBank (acc. No. D88637). 
Table 2. Nucleotide variations of Cyt b gene of local swamp buffalo based on population

\begin{tabular}{|c|c|c|c|c|c|c|c|c|c|c|c|c|c|}
\hline \multirow{3}{*}{ Population } & \multirow{2}{*}{\multicolumn{2}{|c|}{ Conserved Sites }} & \multirow{2}{*}{\multicolumn{2}{|c|}{ Variables }} & \multirow{2}{*}{\multicolumn{2}{|c|}{$\mathrm{Pi}$}} & \multirow{2}{*}{\multicolumn{2}{|c|}{$\mathrm{s}$}} & \multicolumn{4}{|c|}{ Substitution } & \multirow{3}{*}{$\mathrm{Hd}$} \\
\hline & & & & & & & & & \multicolumn{2}{|c|}{ Transition } & \multicolumn{2}{|c|}{ Transversion } & \\
\hline & $\Sigma$ & $\%$ & $\Sigma$ & $\%$ & $\Sigma$ & $\%$ & $\Sigma$ & $\%$ & $\Sigma$ & $\%$ & $\Sigma$ & $\%$ & \\
\hline BK & 861 & 99.42 & 5 & 0.58 & 0 & 0 & 5 & 0.58 & 3 & 0.35 & 2 & 0.23 & 0.4545 \\
\hline $\mathrm{BD}$ & 863 & 99.65 & 3 & 0.35 & 0 & 0 & 3 & 0.35 & 2 & 0.23 & 1 & 0.12 & 0.1818 \\
\hline KL & 862 & 99.54 & 4 & 0.46 & 1 & 0.12 & 3 & 0.35 & 3 & 0.35 & 1 & 0.12 & 0.6703 \\
\hline KN & 861 & 99.42 & 5 & 0.58 & 3 & 0.35 & 2 & 0.23 & 3 & 0.35 & 2 & 0.23 & 0.8095 \\
\hline TR & 864 & 99.77 & 2 & 0.23 & 0 & 0 & 2 & 0.23 & 1 & 0.12 & 1 & 0.12 & 0.1818 \\
\hline NTB & 864 & 99.77 & 2 & 0.23 & 0 & 0 & 2 & 0.23 & 1 & 0.12 & 1 & 0.12 & 0.1818 \\
\hline BTN & 864 & 99.77 & 2 & 0.23 & 0 & 0 & 2 & 0.23 & 1 & 0.12 & 1 & 0.12 & 0.3333 \\
\hline NAD & 862 & 99.54 & 4 & 0.46 & 0 & 0 & 4 & 0.46 & 3 & 0.35 & 1 & 0.12 & 0.8000 \\
\hline All samples & 857 & 98.96 & 9 & 1.04 & 3 & 0.35 & 6 & 0.69 & 7 & 0.81 & 2 & 0.23 & 0.6590 \\
\hline
\end{tabular}

Note: $\mathrm{Pi}=$ parsimony-informative sites; $\mathrm{s}=$ singleton sites; $\mathrm{Hd}=$ haplotype diversity; $\mathrm{BK}=\mathrm{Bombana}$ Island; $\mathrm{BD}=\mathrm{Bombana} \mathrm{Mainland}$; $\mathrm{KL}=\mathrm{Kolaka}$; $\mathrm{KN}=$ Konawe; TR= North Toraja; NTB= West Nusa Tenggara; BTN= Banten; NAD= Nanggroe Aceh Darussalam.

\begin{tabular}{|c|c|c|c|c|c|c|c|c|c|}
\hline \multirow{4}{*}{ Samples } & \multicolumn{9}{|c|}{ Nucle atide sites } \\
\hline & & & & 2 & 3 & 4 & 4 & 6 & 6 \\
\hline & & 1 & 5 & 6 & 8 & 1 & 7 & 3 & 7 \\
\hline & 6 & 2 & 7 & 2 & 1 & 7 & 2 & 1 & 8 \\
\hline D88637* & A & $\mathrm{C}$ & $\mathrm{C}$ & G & G & A & $\mathrm{T}$ & $\mathrm{C}$ & $\mathrm{C}$ \\
\hline BK6 & . & . & $\mathbf{T}$ & . & $\mathrm{C}$ & G & . & . & . \\
\hline BK7 & . & . & $\mathbf{T}$ & . & $\mathrm{C}$ & G & . & . & . \\
\hline BK8 & . & . & $\mathbf{T}$ & . & $\mathrm{C}$ & G & . & . & . \\
\hline BK9 & . & . & $\mathbf{T}$ & . & $\mathrm{C}$ & G & . & . & . \\
\hline BK12 & G & . & $\mathbf{T}$ & . & $\mathrm{C}$ & G & . & . & . \\
\hline BK14 & . & . & $\mathbf{T}$ & & $\mathrm{C}$ & G & . & . & . \\
\hline BK15 & . & . & $\mathbf{T}$ & & $\mathrm{C}$ & G & . & . & . \\
\hline BK18 & . & G & $\mathbf{T}$ & . & $\mathrm{C}$ & G & . & . & . \\
\hline BK21 & . & . & $\mathbf{T}$ & . & $\mathrm{C}$ & G & . & . & . \\
\hline BK24 & . & . & $\mathbf{T}$ & & $\mathrm{C}$ & G & . & . & . \\
\hline BK28 & . & . & $\mathbf{T}$ & & $\mathrm{C}$ & G & . & . & . \\
\hline BD1 & . & . & $\mathbf{T}$ & . & $\mathrm{C}$ & G & . & . & . \\
\hline BD4 & . & . & $\mathbf{T}$ & . & $\mathrm{C}$ & G & . & . & . \\
\hline BD7 & . & . & $\mathbf{T}$ & . & $\mathrm{C}$ & $\mathrm{G}$ & . & . & . \\
\hline BD9 & . & . & $\mathbf{T}$ & & $\mathrm{C}$ & G & . & . & . \\
\hline BD11 & . & . & $\mathbf{T}$ & & $\mathrm{C}$ & $\mathrm{G}$ & . & . & . \\
\hline BD13 & . & . & $\mathbf{T}$ & & $\mathrm{C}$ & G & . & . & . \\
\hline BD14 & . & . & $\mathbf{T}$ & . & $\mathrm{C}$ & G & . & . & . \\
\hline BD15 & . & . & $\mathbf{T}$ & . & $\mathrm{C}$ & G & . & . & . \\
\hline BD19 & . & . & $\mathbf{T}$ & . & $\mathrm{C}$ & G & . & . & . \\
\hline BD25 & . & . & $\mathbf{T}$ & . & $\mathrm{C}$ & G & . & . & . \\
\hline KL1 & . & . & . & . & $\mathrm{C}$ & G & . & . & . \\
\hline KL2 & . & . & . & . & $\mathrm{C}$ & G & . & . & . \\
\hline KL3 & . & . & . & A & $\mathrm{C}$ & G & . & . & . \\
\hline KL4 & . & . & $\mathbf{T}$ & . & $\mathrm{C}$ & $\mathrm{G}$ & . & . & . \\
\hline KL5 & . & . & $\mathbf{T}$ & . & $\mathrm{C}$ & G & . & . & . \\
\hline KL6 & . & . & $\mathbf{T}$ & . & $\mathrm{C}$ & G & . & . & . \\
\hline KL7 & . & . & . & . & $\mathrm{C}$ & G & . & . & . \\
\hline KL8 & . & . & . & . & C & G & . & . & . \\
\hline KL9 & . & . & $\mathbf{T}$ & . & C & G & . & . & . \\
\hline KL10 & . & . & $\mathbf{T}$ & . & $\mathrm{C}$ & G & . & . & . \\
\hline KL11 & . & . & $\mathbf{T}$ & . & C & G & . & . & . \\
\hline KLB1 & . & . & . & . & $\mathrm{C}$ & G & . & . & . \\
\hline KLB2 & . & . & . & . & C & G & . & . & . \\
\hline KN1 & . & G & $\mathbf{T}$ & . & $\mathrm{C}$ & G & . & $\mathrm{T}$ & . \\
\hline $\mathrm{KN} 2$ & . & . & $\mathbf{T}$ & . & $\mathrm{C}$ & G & . & . & . \\
\hline KN3 & . & . & $\mathbf{T}$ & . & $\mathrm{C}$ & G & . & $\mathrm{T}$ & . \\
\hline KN4 & . & G & $\mathbf{T}$ & . & C & G & . & $\mathrm{T}$ & . \\
\hline KN5 & . & . & $\mathbf{T}$ & . & C & G & . & $\mathrm{T}$ & . \\
\hline KN6 & . & G & $\mathbf{T}$ & . & C & G & . & $\mathrm{T}$ & . \\
\hline KN7 & . & . & $\mathbf{T}$ & . & C & G & . & $\mathrm{T}$ & . \\
\hline KN8 & . & . & . & . & C & G & . & . & . \\
\hline KN9 & . & . & . & . & $\mathrm{C}$ & G & . & . & . \\
\hline KN10 & . & . & . & . & C & G & . & . & . \\
\hline KN11 & . & . & $\mathbf{T}$ & . & C & G & . & . & . \\
\hline KN12 & . & . & $\mathbf{T}$ & . & C & G & . & $\mathrm{T}$ & . \\
\hline KN13 & . & . & . & . & $\mathrm{C}$ & G & . & . & . \\
\hline KN14 & $\cdot$ & & $\mathbf{T}$ & . & C & G & . & $\mathrm{T}$ & \\
\hline
\end{tabular}

\begin{tabular}{|c|c|c|c|c|c|c|c|c|c|}
\hline \multirow{4}{*}{ Samples } & \multicolumn{9}{|c|}{ Nucle atide sites } \\
\hline & & & & 2 & 3 & 4 & 4 & 6 & 6 \\
\hline & & 1 & 5 & 6 & 8 & 1 & 7 & 3 & 7 \\
\hline & 6 & 2 & 7 & 2 & 1 & 7 & 2 & 1 & 8 \\
\hline TRB1 & . & . & . & . & $\mathrm{C}$ & $\mathrm{G}$ & . & . & . \\
\hline TRB2 & . & . & . & . & $\mathrm{C}$ & G & . & . & . \\
\hline TRB3 & . & . & . & . & $\mathrm{C}$ & G & . & . & . \\
\hline TRB4 & . & . & . & . & $\mathrm{C}$ & G & . & . & . \\
\hline TRB5 & . & . & . & . & $\mathrm{C}$ & G & . & . & . \\
\hline TRP6 & . & . & . & . & $\mathrm{C}$ & G & . & . & . \\
\hline TRP8 & . & . & . & . & $\mathrm{C}$ & G & . & . & . \\
\hline TRT13 & . & . & . & . & $\mathrm{C}$ & G & . & . & . \\
\hline TRT14 & . & . & . & . & $\mathrm{C}$ & $\mathrm{G}$ & . & . & . \\
\hline TRT15 & . & . & . & . & $\mathrm{C}$ & G & . & . & . \\
\hline NTB1 & . & . & . & . & $\mathrm{C}$ & G & . & . & . \\
\hline NTB2 & . & . & . & . & $\mathrm{C}$ & G & . & . & . \\
\hline NTB3 & . & . & . & . & $\mathrm{C}$ & G & . & . & . \\
\hline NTB5 & . & . & . & . & $\mathrm{C}$ & G & . & . & . \\
\hline NTB6 & . & . & . & . & $\mathrm{C}$ & G & . & . & . \\
\hline NTB7 & . & . & . & . & $\mathrm{C}$ & G & . & . & . \\
\hline NTB8 & . & . & . & . & $\mathrm{C}$ & G & . & . & . \\
\hline NTB9 & . & . & . & . & $\mathrm{C}$ & G & . & . & . \\
\hline NTB10 & . & . & . & . & $\mathrm{C}$ & G & . & . & . \\
\hline NTB11 & . & . & . & . & $\mathrm{C}$ & G & . & . & . \\
\hline BTN1 & . & . & . & . & $\mathrm{C}$ & G & . & . & . \\
\hline BTN2 & . & . & . & . & $\mathrm{C}$ & G & . & . & . \\
\hline BTN3 & . & . & . & . & $\mathrm{C}$ & G & . & . & . \\
\hline BTN4 & . & . & . & . & $\mathrm{C}$ & G & . & . & . \\
\hline BTN5 & . & . & . & . & $\mathrm{C}$ & G & . & . & . \\
\hline NAD1 & . & . & . & . & $\mathrm{C}$ & G & . & . & . \\
\hline NAD2 & . & . & . & . & $\mathrm{C}$ & G & . & . & . \\
\hline NAD3 & . & . & . & . & $\mathrm{C}$ & G & . & . & . \\
\hline NAD9 & . & . & . & . & $\mathrm{C}$ & G & $\mathrm{C}$ & . & . \\
\hline NAD10 & . & . & . & . & $\mathrm{C}$ & G & & . & $\mathrm{T}$ \\
\hline
\end{tabular}

Figure 3. The nucleatide position in the variable sites of Cyt b genes of Indoesian swamp buffaloes. ${ }^{*}$ Sequence from GenBank (acc. No. D88637); BK= Bombana Island; BD= Bombana mainland; KL: Kolaka; KN= Konawe; TR= North Toraja; NTB= West Nusa Tenggara; BTN: Banten; NAD= Nanggroe Aceh Darussalam. 
Konawe population; meanwhile the lowest was found 1 site $(0.12 \%)$ in Kolala population. The parsimony site was not found in Bombana Island, Bombana mainland, North Toraja, NTB, Banten, and NAD populations, meanwhile the highest singleton was found 5 sites $(0.58 \%)$ in Bombana Island population, and the lowest one was found 2 sites $(0.23 \%)$ in Konawe, North Toraja, NTB, Banten, and NAD populations. The nucleotide variations were caused by transition and transversion substitutions of the nucleotide base. Based on the distribution of all samples, it was identified $7(0.81 \%)$ transition substitution sites in the $6,417,262,57,631,678$, and 472 nucleotides, and $2(0.23 \%)$ transversion substitution sites in the 12 and 381 nucleotides.

\section{Genetic Distance}

Genetic distance was calculated based on the Kimura-2 parameter method (Tamura et al., 2013). The average genetic distances among eight Indonesian swamp-buffalo populations were at the range from 0.0000-0.0022 (Table 3). The closest genetic distance was identified among North Toraja, NTB, and Banten populations (0.0000), meanwhile the farthest was between Konawe and NAD populations (0.0022).

\section{Phylogenetic Tree Reconstruction}

The results of the phylogenetic tree reconstruction (Figure 4) showed that eight Indonesian swamp-buffalo populations in this study were grouped into two major clusters. The first cluster amounted to 42 samples $(53.16 \%)$ consisting of the populations of NAD $(11.90 \%)$, Banten (11.90\%), North Toraja (23.81\%), NTB (23.81\%), Kolaka (16.68\%), Konawe $(9.52 \%)$, and buffalo out of the groups from GenBank (2.38\%). Meanwhile the second cluster amounted to 37 samples (46.84\%) which all samples were from Southeast Sulawesi, including Bombana Island $(29.73 \%)$, Bombana mainland $(27.03 \%)$, Kolaka $(16.21 \%)$, and Konawe (27.03\%). In the second cluster, two subclusters were formed consisting of Bombana Island and Konawe populations.

\section{Haplotype}

The results of the haplotype analysis based on the distribution of all samples (including sequences from GenBank), were found 10 haplotypes with the haplotype diversity value of 0.6590 . Haplotype percentages of eight Indonesian swamp buffaloe populations (Table 4) showed that the haplotype 5 had the highest individual percentage $(48.10 \%)$ consisting of Kolaka $(15.78 \%)$, Konawe $(10.53 \%)$, North Toraja (26.32\%), NTB $(26.32 \%)$, Banten $(13.16 \%)$, and NAD populations (7.89\%). Further, the haplotype 2 was the second higher percentage $(34.18 \%)$ consisting of Bombana Island (33.33\%), Bombana mainland (37.04\%), Kolaka (22.22\%), and Konawe populations $(7.41 \%)$. There were some buffaloe populations that formed separate haplotypes (H_), with individual percentages ranges from 1.27 to $6.33 \%$, namely Bombana Island (H_3 and H_4), Kolaka (H_6), Konawe (H_7 and $\left.\mathrm{H} \_8\right)$, and NAD populations $\left(\overline{\mathrm{H}} \_9\right.$ and H_10). The sequences of Cyt $b$ gene of each haplotype are presented in Figure 5. Meanwhile, MedianJoining network of haplotypes of eight Indonesian swamp buffalo populations is presented in Figure 6.

\section{DISCUSSION}

\section{Nucleotide Variations}

A total of 78 Cyt b genes sequences of local swamp buffaloes from 8 different populations were observed in this study. Previous studies reported that from 17 Cyt b gene sequences of Indonesian swamp buffaloes, $80.13 \%$ were found to be conserved sites and $19.87 \%$ were found to be variable sites (Sukri et al., 2014). In the present study, it was found 856 (98.96\%) conservation sites and $9(1.04 \%)$ variable sites. Cyt b genes sequences of Indonesian swamp buffaloes in this study were more conservative than those reported by Sukri et al. (2014) that found lower variable sites. The use of different Cyt $b$ genes references from the GenBank influenced the results. Cyt $b$ gene sequence reference used in this study was from swamp buffalo (Bubalus bubalis carabanesis); meanwhile Sukri et al. (2014) used Cyt b genes sequences reference from African buffalo (Syncerus caffer).

The variable sites found in this study consists of $3(0.35 \%)$ parsimony-informative sites and $3(0.35 \%)$ singleton sites. The parsimony-informative site was only found in Kolaka $(0.12 \%)$ and Konawe populations $(0.35 \%)$, meanwhile singleton site was found in all populations. The nucleotide variations of Cyt $b$ gene of local swamp buffaloes in this study were lower than that

Table 3. Average (below diagonal) and standard deviation (above diagonal) of genetic distance of eight Indonesian swamp buffalo populations

\begin{tabular}{ccccccccc}
\hline Populations & BK & BD & KL & KN & TR & NTB & BTN & NAD \\
\hline BK & - & 0.0005 & 0.0005 & 0.0004 & 0.0000 & 0.0005 & 0.0005 & 0.0005 \\
BD & 0.0002 & - & 0.0000 & 0.0000 & 0.0000 & 0.0000 & 0.0000 & 0.0000 \\
KL & 0.0009 & 0.0007 & - & 0.0005 & 0.0006 & 0.0006 & 0.0006 & 0.0006 \\
KN & 0.0014 & 0.0012 & 0.0016 & - & 0.0013 & 0.0013 & 0.0013 & 0.0013 \\
TR & 0.0014 & 0.0012 & 0.0006 & 0.0017 & - & 0.0000 & 0.0000 & 0.0000 \\
NTB & 0.0014 & 0.0012 & 0.0006 & 0.0017 & 0.0000 & - & 0.0000 & 0.0000 \\
BTN & 0.0014 & 0.0012 & 0.0006 & 0.0017 & 0.0000 & 0.0000 & - & 0.0000 \\
NAD & 0.0018 & 0.0016 & 0.0011 & 0.0022 & 0.0005 & 0.0005 & 0.0005 & - \\
\hline
\end{tabular}

Note: $\mathrm{BK}=$ Bombana Island; $\mathrm{BD}=$ Bombana Mainland; $\mathrm{KL}=$ Kolaka; $\mathrm{KN}=$ Konawe; $\mathrm{TR}=$ North Toraja; NTB= West Nusa Tenggara; $\mathrm{BTN}=\mathrm{Banten}$; NAD= Nanggroe Aceh Darussalam. 


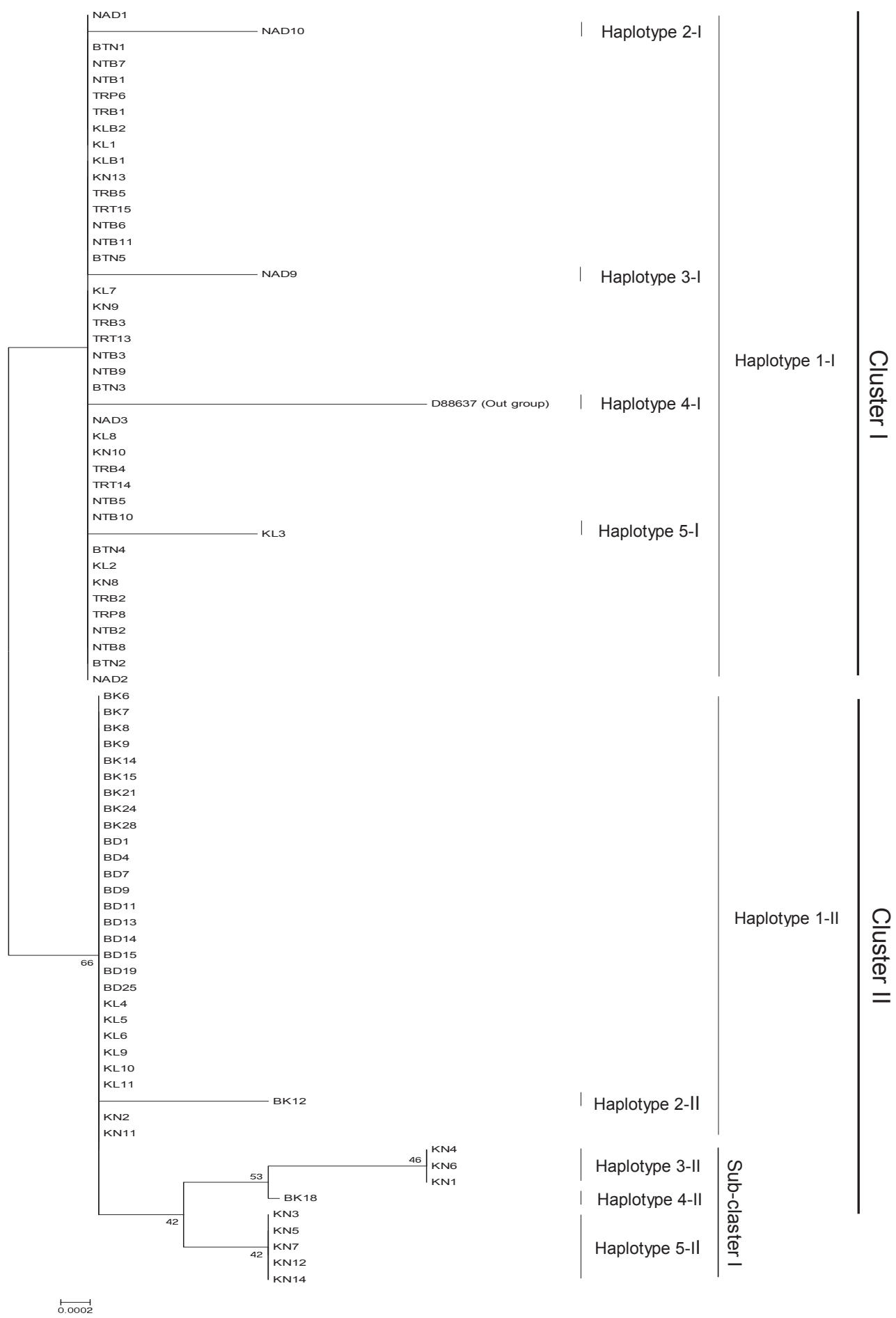

Figure 4. Phylogenetic tree of eight Indonesian swamp buffalo populations based on Cyt $\mathrm{b}$ gene sequences, including sequence from GenBank (acc. No. D88637); BK= Bombana Island; BD= Bombana mainland; KL: Kolaka; KN= Konawe; TR= North Toraja; NTB= West Nusa Tenggara; BTN: Banten; NAD= Nanggroe Aceh Darussalam.

of cattle. In Romanian cattle populations, 95 polymorphic sites were detected consisted of 73 parsimony-informative sites and 21 singleton sites (Xuan et al., 2010), meanwhile in Ethiopian cattle populations, 18 polymorphic sites were detected consisting of seven parsimonyinformative sites and 11 singleton sites (Tarekegn et al., 2018).

The positions of the variable site were found in the $6,12,57,262,831,417,472,631$, and 678 nucleotides
(Figure 4). In the 57 nucleotide, $\mathrm{C}$ base changed to $\mathrm{T}$. This mutation (g.57C $>\mathrm{T}$ ) was only found in the Cyt b gene sequences of swamp buffaloes from Southeast Sulawesi, especially Bombana Island and Bombana mainland populations. This nucleotide mutation was found in all swamp buffaloes from the Bombana Island and Bombana mainland populations. The site of g.57C $>\mathrm{T}$ could be claimed as a specific nucleotide site (geographic origin) to the Island Bombana and main- 
Table 4. Percentage of haplotypes based on Cyt b gene sequences of eight Indonesian swamp buffalo populations

\begin{tabular}{|c|c|c|c|c|c|c|c|c|c|c|c|}
\hline \multirow{2}{*}{ Haplotypes } & \multicolumn{9}{|c|}{ Populations } & \multirow{2}{*}{ Total } & \multirow{2}{*}{$\%$} \\
\hline & $\mathrm{Bbc}$ & $\mathrm{BK}$ & $\mathrm{BD}$ & KL & $\mathrm{KN}$ & TR & NTB & BTN & NAD & & \\
\hline 1 & 1 & - & - & - & - & - & - & - & - & 1 & 1.27 \\
\hline 2 & - & 9 & 10 & 6 & 2 & - & - & - & - & 27 & 34.18 \\
\hline 3 & - & 1 & - & - & - & - & - & - & - & 1 & 1.27 \\
\hline 4 & - & 1 & - & - & - & - & - & - & - & 1 & 1.27 \\
\hline 5 & - & - & - & 6 & 4 & 10 & 10 & 5 & 3 & 38 & 48.10 \\
\hline 6 & - & - & - & 1 & - & - & - & - & - & 1 & 1.27 \\
\hline 7 & - & - & - & - & 3 & - & - & - & - & 3 & 3.80 \\
\hline 8 & - & - & - & - & 5 & - & - & - & - & 5 & 6.33 \\
\hline 9 & - & - & - & - & - & - & - & - & 1 & 1 & 1.27 \\
\hline 10 & - & - & - & - & - & - & - & - & 1 & 1 & 1.27 \\
\hline $\mathrm{N}$ & 1 & 11 & 10 & 13 & 14 & 10 & 10 & 5 & 5 & 79 & 100.00 \\
\hline
\end{tabular}

Note: Bbc: Out group Buffalo (GenBank accession number: D88637); BK= Bombana Island; BD= Bombana Mainland; KL= Kolaka; KN= Konawe; TR= North Toraja; NTB= West Nusa Tenggara; BTN= Banten; NAD= Nanggroe Aceh Darussalam.

land Bombana populations. The specific nucleotide site with the same nucleotide position (g.57C $>\mathrm{T}$ ) was also found in several other swamp buffaloes from Southeast Sulawesi, consisted of Kolaka (6 individuals) and Konawe (10 individuals) populations. The emergence of the specific nucleotides sites was an influence of forming a new population (founder effect) as a result of genetic drift which was a situation where a small number of individuals move to a new place and to form a new population. The founder effect was an example of the population bottleneck which was a situation where the number of parents in the population becomes very small for one generation or more (Nicholas, 2010).

The nucleotide variations could be due to the mutations of nucleotide bases. The results of previous studies reported that there were 4 transition substitutions $(\mathrm{C}>\mathrm{T})$ in $\mathrm{Cyt} \mathrm{b}$ gene sequence of swamp buffaloes in Asian mainland (Thailand and Peninsular Malaysia), but transversion substitution was not found (Lau et al., 1998). Also, Kim et al. (2013) found fifteen transition substitutions in the Korean cattle from fifteen polymorphic sites identified. In contrast, in the Pakistani river buffaloes (Nili-Ravi, Kundi) there were 79 transition substitutions (g.33A $>\mathrm{G}$ and g.46C $>\mathrm{T}$ ) and 43 transver- sion substitutions with a ratio of 1.83: 1 (Saif et al., 2012). In this study, $7(0.81 \%)$ transitional substitution and $2(0.23 \%)$ transversion substitution were found in Cyt $\mathrm{b}$ gene sequence of Indonesian swamp buffaloes with a ratio of 6.67: 1. The nucleotide substitutions in this study were silent mutations because it only occurred in the third codon translating the same amino acid even though it has different DNA sequences (Saif et al., 2012).

\begin{tabular}{|c|c|c|c|c|c|c|c|c|c|}
\hline \multirow{4}{*}{ Haplotype } & \multicolumn{9}{|c|}{ Nucleotide position } \\
\hline & & & & 2 & 3 & 4 & 4 & 6 & 6 \\
\hline & & 1 & 5 & 6 & 8 & 1 & 7 & 3 & 7 \\
\hline & 6 & 2 & 7 & 2 & 1 & 7 & 2 & 1 & 8 \\
\hline H_1 & A & $\mathrm{C}$ & $\mathrm{C}$ & G & G & $\mathrm{A}$ & $\mathrm{T}$ & $\mathrm{C}$ & $\mathrm{C}$ \\
\hline H_2 & . & . & $\mathrm{T}$ & . & $\mathrm{C}$ & G & . & . & . \\
\hline $\mathrm{H}_{-}^{-} 3$ & G & . & $\mathrm{T}$ & . & $\mathrm{C}$ & G & . & . & . \\
\hline H_4 & . & G & $\mathrm{T}$ & . & $\mathrm{C}$ & G & . & . & . \\
\hline H_5 & . & . & r. & . & C & G & . & . & . \\
\hline H_6 & . & & & A & C & G & . & & . \\
\hline $\mathrm{H}_{-}^{-} 7$ & . & G & $\mathrm{T}$ & 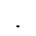 & C & G & . & $\mathrm{T}$ & . \\
\hline H_8 & . & . & $\mathrm{T}$ & . & $\mathrm{C}$ & G & & $\mathrm{T}$ & . \\
\hline H_9 & . & . & . & . & C & G & C & . & . \\
\hline H_10 & . & . & . & . & C & G & . & . & $\mathrm{T}$ \\
\hline
\end{tabular}

Figure 5. The sequences of haplotypes of eight Indonesian swamp-buffalo populations

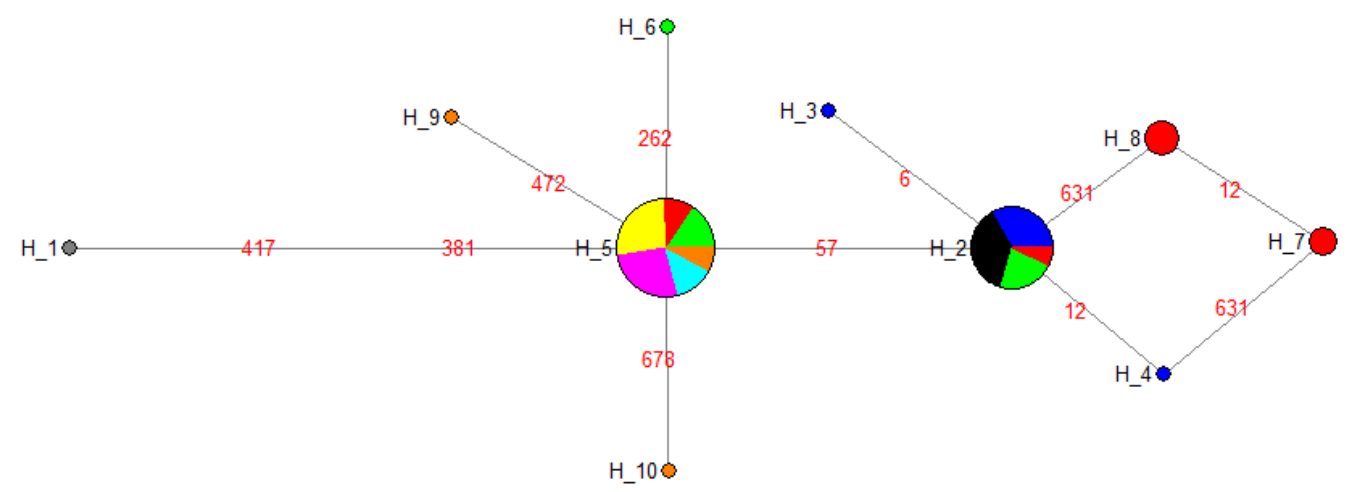

Figure 6. The Median-Joining network of Cyt b gene haplotype of eight Indonesian swamp-buffalo populations. Color key: $\square=$ D88637 (out grop); $\square=$ NAD; $\square=$ Banten; $\square=$ NTB; $\square=$ North Toraja; $\square=$ Bombana Island; $\boldsymbol{\square}=$ Bombana mainland; $\square=$ Kolaka; $\square=$ Konawe . 
A higher transition substitution than transversion was a characteristic of the Cyt b gene (Avise, 1994). Transition substitutions were found in the nucleotide position of 6th (g.6A>G), 417th (g.417A $>\mathrm{G})$, 262th (g.262G $>A)$, 57th (g.57C $>$ T), 631th (g.631C $>\mathrm{T})$, 678th (g.678C $>\mathrm{T})$, and 472 th $(\mathrm{g} .472 \mathrm{~T}>\mathrm{C})$, meanwhile transversion substitutions were found in the nucleotide position of 12th (g.12C>G) and 381th (g.381G>C). The substitution between pyrimidine base ( $T$ and $C$ ) was higher than the substitution between purine bases (A and $G$ ). The results of this study support the statement of Avise (1994) and Xuan et al. (2010).

\section{Phylogeography}

In this study, there were identified two major clusters from eight Indonesian swamp-buffalo populations based on their topology phylogenetic trees (Figure 4). This indicates that the Indonesian swamp-buffalo populations have two different maternal lineages. The formation of these lineages was possibly related to the entry path of swamp buffaloes from their domestication center. Molecular genetic studies confirmed that swamp buffaloes were surmised of becoming domesticated in South China (Lei et al., 2007) or in the border regions between South China and North Vietnam (Wang et al., 2017) or Indo-Chinese and North Thailand Southeast Asia (Lau et al., 1998; Zhang et al., 2011). Swamp buffaloes from the domestication centers migrated to Indonesia through two routes. The first route was through Vietnam, Thailand, the Malay Peninsula, Sumatera, Java, Sulawesi, and Nusa Tenggara; meanwhile the second route was through China, Taiwan, Philippines, and Kalimantan (Zhang et al., 2011; Wang et al., 2017).

The phylogenetic tree (Figure 4) was shown that the first cluster (lineage I) consisted of NAD, Banten, NTB, and North Toraja populations and also a portion of Kolaka and Konawe populations. This indicated that they came from the same maternal lineage. Meanwhile, all of individual swamp buffaloes in the second cluster (lineage II) originated from Southeast Sulawesi consisted of Bombana Island, Bombana mainland, Kolaka, and Konawe populations. This indicated that swamp buffaloes spreading in various regions of Southeast Sulawesi originated from the same maternal lineage and they were different from the other swamp-buffaloe populations in Indonesia. The results of previous studies also found two different maternal lineages in Chinese native swamp buffalo based on DNA Cyt $b$ gene and D-loop of mitochondrial (Lei et al., 2011; Yue et al., 2013), and swamp buffalo in Southeast Asia based on mitochondrial D-loop (Lei et al., 2007).

The highest genetic similarity $(100 \%)$ was found between Banten, NTB, and North Toraja populations with a genetic similarity were $100 \%$; meanwhile the furthest one was between Konawe and NAD populations, which they had the farthest geographical distance from all populations. The genetic distances among Indonesian swamp-buffaloe populations in this study were relatively closer compared to the swamp-buffalo populations in Southeast Asia based on D-loop sequences of mitochon- drial DNA at the ranges of -0.0041 to 0.0486 (Lau et al., 1998). Closer genetic distance (0.0000-0.0005) based on Cyt $b$ gene sequence of mitochondrial DNA was detected in wild Java Banteng populations in the four different national parks (Qiptiyah et al., 2019). Meanwhile, further genetic distances based on Cyt $b$ gene sequence of mitochondrial DNA was detected in Ethiopian native cattle populations, at the ranges of 0.00010 to 0.12446 (Tarekegn et al., 2018).

The genetic relationships among Indonesian swamp-buffaloe populations in this study were not correlated with the geographical distance of their habitats. For instance, geographical distance between North Toraja and Banten populations was further than that of geographical distance between North Toraja and Kolaka populations, but genetic distance between North Toraja and Banten populations was closer than that of between North Toraja and Kolaka populations, although swamp-buffalo populations of North Toraja, Kolaka, and Konawe originated from the same Island (Sulawesi Island). That a number of local swamp buffaloes may have a fairly close genetic relationships, and others have far genetic relationships. Genetic relationships level was often found being uncorrelated with the geographical distance of buffalo habitat. This result might be related to the fact that there were some populations that were more open because transportation routes were easily accessible so that it made easier for buffalo migration (Anggraeni et al., 2011).

Haplotypes and nucleotides diversities are alternative measures of mitochondrial DNA variations (Yindee et al., 2010). In this study, 10 haplotypes were found with haplotypes diversity of 0.6590 from 79 Cyt $\mathrm{b}$ genes sequences of swamp buffalo (including buffalo from GenBank). The number of haplotypes of Cyt $\mathrm{b}$ gene sequences of eight Indonesian swamp-buffaloe populations in this study was higher than that reported by Saputra et al. (2013) that they only found three haplotypes of seven Indonesian swamp-buffaloe populations based on the 21 Cytochrome Oxidase subunit I (COI) gene sequences. The higher haplotypes number of Cyt $\mathrm{b}$ gene sequences of Indonesian swamp buffalo was reported by Sukri et al. (2014) as many as 16 haplotypes from 17 Cyt $b$ gene sequences. Meanwhile, in Thailand swamp buffalo there were 14 haplotypes from $62 \mathrm{Cyt} \mathrm{b}$ gene sequences (Yindee et al., 2010).

Based on the results of the haplotype analysis and the median-joining network of haplotypes (Figure 5), two major haplotype groups were identified in the Indonesian swamp buffalo, namely haplotype 5 (H_5) and haplotype 2 (H_2). The major haplotypes act as radiating nodes for many other haplotypes (Kathiravan et al., 2011). H_5 is the haplotype as a representative of the Indonesian swamp buffalo which is widespread in all Indonesia regions, both in NAD, Java, and NTB or Sulawesi, especially North Toraja and partly Kolaka and Konawe. Meanwhile, H_2 is a haplotype as a representative of Southeast Sulawesi swamp buffalo. The haplotypes in the Bombana Island region and Bombana mainland region were specific breeds because they were only found in the second cluster and H_2 (see Figures 4 and 5). This condition was different from Konawe and 
Kolaka haplotypes which could be found in the first clusters and H_5 and also in the second clusters and H_2. Therefore, the haplotypes in the population of Bombana Island region and Bombana mainland region were feasible to be grouped as different breeds/strains from the other populations. The populations of swamp buffaloes in Bombana Island and Bombana mainland have the possibility of originating from an expansion of H_5 especially from the populations either from Kolaka or Konawe buffaloes migrating to Bombana Regency and experienced a mutation.

The other seven haplotypes (H-3, H_4, and H_6 to H_10) were expansion haplotypes from two major haplotypes. The formation of separate haplotypes from the major haplotype indicates the existence of groups or strains of Indonesian swamp buffalo that were genetically different. According to Coroian et al. (2015), the formation of a haplotype indicates that the population isolated from the viewpoint of geography and reproduction. Based on the polymorphic sites number (Table 2) and percentage of haplotypes (Tabel 4), it can be claimed that the population of Southeast Sulawesi swamp buffaloes was relatively higher than the populations of buffaloes from Aceh, Banten, NTB, and North Toraja, where the highest polymorphic site haplotypes number were found in the populations of Konawe and Bombana Island.

\section{CONCLUSION}

The results of the study concluded that eight Indonesian local swamp-buffaloe populations were grouped into two main clusters. Bombana Island and Bombana mainland populations were specific breeds because they were only found in the second cluster and also have specific nucleotides sites on the 57 nucleotides which $C$ base changed to $T$. The results of this study were useful in formulating the program of conservation and utilization of Indonesian buffalo genetic resources, especially in the buffalo population with specific breeds.

\section{CONFLICT OF INTEREST}

We certify that there is no conflict of interest with any financial, personal, or other relationships with other people or organizations related to the material discussed in the manuscript.

\section{ACKNOWLEDGEMENT}

Authors thank very much to the Ministry of Research, Technology and Higher Education of Indonesia Republic for their contribution to support the financing of this research through the Doctoral Dissertation Research Grant 2018.

\section{REFERENCES}

Amin, M., E. Suarsini, I. Azmi, \& A. Gofur. 2016. Phylogenetic analysis of local endemic buffalo (Bubalus bubalis) based on cytochrome $b$ gene in central Indonesia. J. Teknologi (Science and Angineering) 78:393-397. https://doi. org/10.11113/jt.v78.8343
Anggraeni, A., C. Sumantri, L. Praharani, \& E. Andreas. 2011. Genetic distance estimation of local swamp buffaloes through morphology analysis approach. Jurnal Ilmu Ternak dan Veteriner 16: 199-210.

Arif, I.A. \& H.A. Khan. 2009. Molecular markers for biodiversity analysis of wildlife animals: a brief review. Anim. Biodiv. Conserv. 32: 9-17.

Avise, J.C. 1994. Molecular Markers, Natural History and Evolution. Chapman and Hall, New York. https://doi. org/10.1007/978-1-4615-2381-9

Browers, N., J.R. Stauffer \& T.D. Kocher. 1994. Intra and interspecific mitochondrial DNA sequence variation within two species of rock-dwelling Cichlids (Teleostei: Cichlidae) from Lake Malawi, Africa. Mol. Phylogenet. Evol. 3:75-82. https://doi.org/10.1006/mpev.1994.1009

Coroian, C.O., A. Coroian, V. Mireșan, M. Şuteu, C. Lațiu, \& C. Raducu. 2015. New MTCYB haplotypes in Romanian Buffalo. Bulletin UASVM Animal Science and Biotechnologies 72: 142-147. https://doi.org/10.15835/ buasvmcn-asb:11552

[Ditjen PKH] Direktorat Jenderal Peternakan dan Kesehatan Hewan Kementerian Pertanian Republik Indonesia. 2018. Statistika Peternakan dan Kesehatan Hewan 2018. Ditjen PKH Kementan RI, Jakarta.

Doosti, A., P.G. Dehkordi, \& E. Rahimi. 2014. Molecular assay to fraud identification of meat products. J. Food Sci. Technol. 51:148-152. https://doi.org/10.1007/ s13197-011-0456-3

FAOSTAT. 2015. FAO Statistical Yearbook. http://faostat3.fao. org. [25 October 2015].

FAOSTAT. 2018. FAO Statistical Yearbook. http://faostat3.fao. org. [23 July 2019].

Hartatik, T., W.B.P. Putra, S.D. Volkandari, \& Sumadi. 2015. Polymorphism of mtDNA cytochrome $b$ gene of local cattle in Indonesia. J-SustaiN 3:21-24. https://doi.org/10.24910/ jsustain/3.1/2124

Hartatik, T., D. Maharani, J.H.P. Sidadolog, A. Fathoni, \& Sumadi. 2018. Haplotype diversity of partial cytochrome b gene in Kebumen ongole grade cattle. Trop. Anim. Sci. J. 41:8-14. https://doi.org/10.5398/tasj.2018.41.1.8

Hussain, T., M.E. Babar, M.D. Donato, A. Wajid, A. Nadeem, Z. Ahmad, W.A Khan, S.O. Peters, \& I.G. Imumorin. 2018. Phylogeny of Pakistani cattle breeds using mitochondrial cytochrome b gene. Pakistan J. Zool. 50: 2029-2035. https://dx.doi.org/10.17582/journal.pjz/2018.50.6.2029.2035

Kathiravan, P., R.S. Kataria, B.P. Mishra, P.K. Dubey, D.K. Sadana, \& B.K. Joshi. 2011. Population structure and phylogeography of Toda buffalo in Nilgiris throw light on possible origin of aboriginal Toda tribe of South India. J. Anim. Breed. Genet. 128:295-304. https://dx.doi. org/10.1111/j.1439-0388.2011.00921.x

Kim, J.H., M.J. Byun, K. Myung-Jick, S.W. Suh, K. YeoungGyu, C.W. Lee, J. Kyoung-Sub, E.S. Kim, D.J. Yu, W.H. Kim, \& C. Seong-Bok. 2013. mtDNA Diversity and Phylogenetic State of Korean Cattle Breed, Chikso AsianAustralas. J. Anim Sci. 26163-170. http://dx.doi.org/10.5713/ ajas.2012.12499

Lau, C.H, R.D. Drinkwater, K. Yusoff, S.G. Tan, D.J. Hetzel, \& J.S.F. Barker. 1998. Genetic diversity of Asian water buffalo (Bubalus bubalis): mitochondrial DNA D-loop adn cytochorome b sequence variation. Anim. Genet. 29:253264. https://doi.org/10.1046/j.1365-2052.1998.00309.x

Lei, C.Z., W. Zhang, H. Chen, F. Lu, Q.L. Ge, R.Y. Liu, R.H. Dang, Y.Y. Yao, L.B. Yao, Z.F. Lu, \& Z-I. Zhao. 2007. Two maternal lineages revealed by mitochondrial DNA D-loop sequences in Chinese native water buffaloes (Bubalus bubalis). Asian-Australas. J. Anim Sci. 20:471-476. https://doi. org/10.5713/ajas.2007.471

Lei, C.Z., C.M. Zhang, S. Weining, M.G. Campana, M.A. Bower, X.M Zhang, L. Liu, X.Y. Lan, \& H. Chen. 2011. 
Genetic diversity of mitochondrial cytochrome $b$ gene in Chinese native buffalo. Anim. Genet. 42:432-436. https:// doi.org/10.1111/j.1365-2052.2011.02174.x

Linacre, A., \& S.S. Tobe. 2011. An overview to the investigative approach to species testing in wildlife forensic science. Investig Genet. Open Access. BioMed Central. 2:1-9 https://doi.org/10.1186/2041-2223-2-2

Nicholas, F.H. 2010. Introduction to Veterinary Genetics. $3^{\text {rd }}$ ed. Wiley-Blackwell Publising Ltd, UK.

Pakpahan, S., W.T. Artama, R. Widayanti, \& G. Suparta. 2016. Genetic Characteristics and relationship in different goat populations of Indonesia based on cytochrome b gene sequences. Asian J. Anim. Sci. 10:29-38. https://doi. org/10.3923/ajas.2016.29.38

Qiptiyah, M., S. Pudyatmoko, A. Widyatmoko, M.A. Imron, \& I. Nurtjahjaningsih. 2019. Cytochrome b mitochondrial DNA characteristic from non-invasive samples of wild population Javan Banteng (Bos javanicus d'Alton, 1823). Biodiversitas 20:350-355. https://doi.org/10.13057/biodiv/ d200207

Rozas, J., P. Librado, J.C. Sanchez-DelBarrio, X. Messeguer, \& R. Rozas. 2010. DNA Sequence Polymorphism Version 5.10.01. http:/www.ub.es/dnasp

Saif, R., M.E. Babar, A.R. Awan, A. Nadeem, A.S. Hashmi, \& T. Hussain. 2012. DNA fingerprinting of Pakistani buffalo breeds (Nili-Ravi, Kundi) using microsatellite and cytochrome b gene markers. Mol. Biol. Rep. 39:851-856. https:// doi.org/10.1007/s11033-011-0808-0

Sambrook, J., \& D.W. Russell. 2001. Molecular Cloning: A Laboratory Manual. $3^{\text {rd }}$ ed. Cold Spring Harbor Laboratory Press, USA.

Saputra, F, Jakaria, \& C. Sumantri. 2013. Genetic variation of mtDNA Cytochrome Oxidase Subunit I (COI) in local swamp buffaloes in Indonesia. Med. Pet. 36:165-170 https://doi.org/10.5398/medpet.2013.36.3.165

Sumantri, C., A. Gunawan, \& A. Anggraeni. 2017. Karakteristik Genetik Kerbau Lokal dan Prospek Pengembangannya. IPB Press, Bogor.

Sukri, A., M. Amin, A. Winaya, \& A. Gofur. 2014. Substitution and haplotype diversity analysis on the partial sequence of the mitochondrial DNA Cyt b of Indonesian Swamp Buffalo (Bubalus bubalis). Biomedich. 3:65-70. https://doi. org/10.14421/biomedich.2014.32.59-63

Tarekegn, G.M., X-Y. Ji, X. Bai, B. Liu, W. Zhang, J. Birungi, A. Djikeng, \& K. Tesfaye. 2018. Variations in mitochondrial cytochrome $b$ region among Ethiopian indigenous cattle populations assert Bos taurus maternal origin and historical dynamics. Asian-Australas. J. Anim Sci. 31:13931400. https://doi.org/10.5713/ajas.17.0596
Talib, C., T. Herawati, \& Hastono. 2014. Strategi peningkatan produktivitas kerbau melalui perbaikan pakan dan genetik. Wartazoa 24: 83-96.

Tamura, K., G. Stecher, D. Peterson, A. Filipski, \& S. Kumar. 2013. MEGA 6: Molecular evolutionary genetics analysis version. Mol. Biol. Evol. 30: 2725-2729. https://doi. org $/ 10.1093 / \mathrm{molbev} / \mathrm{mst} 197$

Wang, S., N. Chen, M.R. Capodiferro, T. Zhang, H. Lancioni, H. Zhang, Y. Miao, V. Chanthakhoun, M. Wanapat, M. Yindee, Y. Zhang, H. Lu, L. Caporali, R. Dang, Y. Huang, X. Lan, M. Plath, H. Chen, J.A. Lenstra, A. Achilli, \& C. Lei. 2017. Whole mitogenomes reveal the history of swamp buffalo: initially shaped by glacial periods and eventually modelled by domestication. Scientific Reports. 7:4708. https://doi.org/10.1038/s41598-017-04830-2

Xuan, T.P, S.E. Georgescu, M.A. Manea, A.O. Hermenean, \& M. Costache. 2010. Genetic diversity and phylogenetic relationship of Romanian cattle breeds inferred from cytochrome $b$ gene partial sequences. Rom. Biotechnol. Lett. 15: 5154-5158.

Yindee, M. 2010. Reproduction and genetic diversity of the swamp buffalo. Acad. Thesis, Utrecht University, Utrecht. ISBN 978-90-393-5399-8. (Chapter 5 - High diversity of the mitochondrial DNA of the Thai swamp buffalo indicates a domestication in Southeast Asia, pp. 63-76). http://igiturarchive.library.uu.nl/dissertations/2011-0121-200244/ Yindee.pdf.

Yue, X-P., R. Li, W-M. Xie, P. Xu, T-C. Chang, L. Liu, F. Cheng, R-F. Zhang, X-Y. Lan, Hong Chen, et al. 2013. Phylogeography and domestication of Chinese swamp buffalo. Plos One 8:1-13. https://doi.org/10.1371/journal. pone. 0056552

Zardoya, R. \& A. Meyer. 1996. Phylogenetic performance mitochondrial protein coding genes in resolving relationship among vertebrate. Mol. Biol. Evol. 13: 933-942.

Zhang, Y., D. Vankan, Y. Zhang, \& J.S.F. Barker. 2011. Genetic differentiation of water buffalo (Bubalus bubalis) populations in China, Nepal and south-east Asia: inferences on the region of domestication of the swamp buffalo. Anim Genet. 42:366-377. https://doi. org/10.1111/j.1365-2052.2010.02166.x

Zhang, Y.I., L.U. Yongfang, M. Yindee, K-Y. Li, H-Y. Kuo, Y-T. Ju, S. Ye, M.D.O. Faruque, Q. Li, Y. Wang et al. 2016. Strong and stable geographic differentiation of swamp buffalo maternal and paternal lineages indicates domestication in the China/Indochina border region. Mol. Ecol. 25: 1530-1550. https://doi: 10.1111/mec.13518 